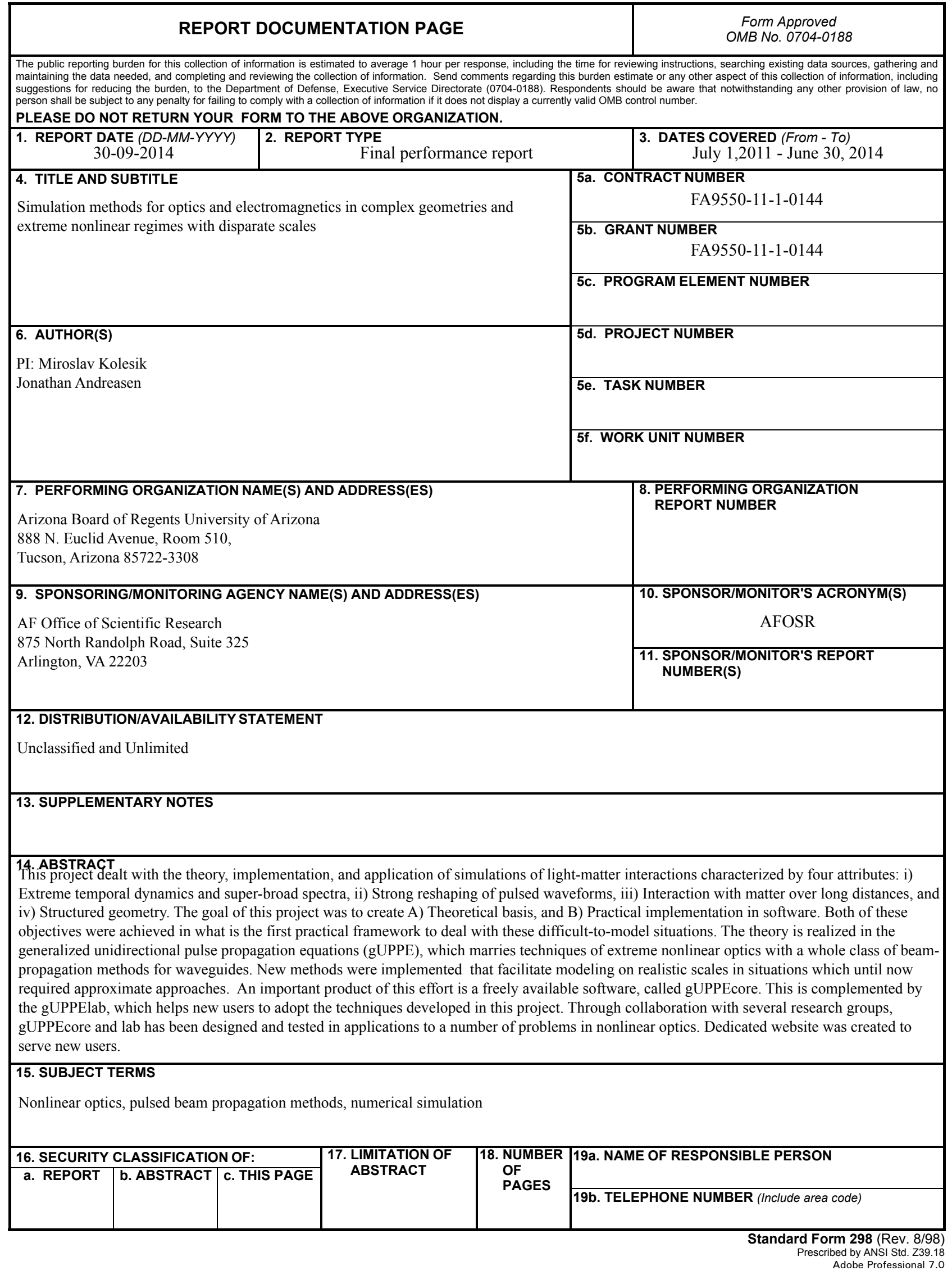




\title{
Contract/Grant Title:
}

Simulation methods for optics and electromagnetics in complex geometries and extreme nonlinear regimes with disparate scales.

\author{
Contract/Grant \#: FA9550-11-1-0144 \\ Principal investigator: Miroslav Kolesik \\ AFOSR program manager: Dr. Arje Nachman \\ Period of performance: 1 July 2011 to 30 June 2014
}

Submission date: 30 Sep. 2014

\section{Contents}

1 Executive Summary $\quad 2$

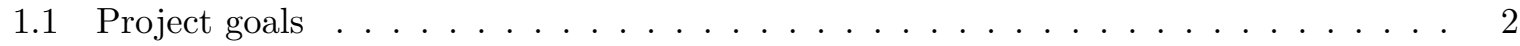

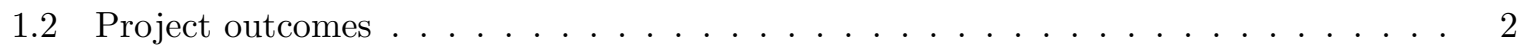

1.3 Broader impact . . . . . . . . . . . . . . . . . . . . . 2

1.4 Personnel Supported . . . . . . . . . . . . . . . . . . . . . 2

1.5 Brief summary of results concerning numerical methods . . . . . . . . . . . . . 3

1.6 Physical results made possible by simulation capabilities developed in this project . 4

$\begin{array}{lll}2 & \text { Technical report } & \mathbf{7}\end{array}$

2.1 Theory of nonlinear pulse propagation in general structured media . . . . . . . . . 7

2.2 New simulation methods for NLO in high-contrast waveguides . . . . . . . . . . . 8

2.3 gUPPEcore and gUPPElab software . . . . . . . . . . . . . . . . . 14

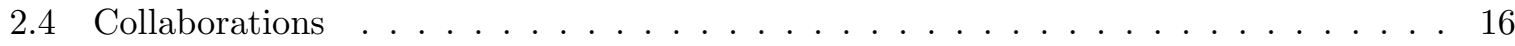

3 Peer-Reviewed Journal Articles $\quad 17$

3.1 Peer-reviewed publications acknowledging the support under this project . . . . . . 17

3.2 Under-review publications acknowledging the support under this project . . . . . . . 18

3.3 Peer-reviewed works utilizing software developed in this project . . . . . . . . 18

4 Dissemination and Outreach $\quad 19$

4.1 Colloquiua, invited talks, and keynote lectures delivered by PI . . . . . . . . . . 19

4.2 Summer-school lectures and short courses delivered by PI . . . . . . . . . . . 20 


\section{Executive Summary}

\section{$1.1 \quad$ Project goals}

This work dealt with the i) theory, ii) implementation, and iii) application of numerical simulations of nonlinear light-matter interactions characterized by the following four attributes:

- Extreme temporal dynamics resulting in super-broad spectra

- Strong spatial reshaping of pulsed waveforms

- Propagation and interaction with matter over long distances

- Structured geometry characterized by strong contrast between constituent materials

The two-fold goal of this project was to create A) Theoretical basis, and B) Practical implementation in software for such regimes. Both of these objectives were achieved in what is the first practical framework to deal with these difficult-to-model situations.

\subsection{Project outcomes}

Peer-reviewed publications. The results of this work have been reported in fourteen peerreviewed publications, twelve of which have appeared or are in press, and two that are presently under review. Moreover, there are seven other published peer-reviewed papers which utilized the software created in this project.

Invited lectures, and summer school courses. The principal investigator delivered one colloquium, one keynote lecture, and eight invited talks, in presenting the theoretical results and the software developed. The PI also taught two summer-school courses and one short course which discussed extensively the theory and algorithms developed.

Free simulation software and dedicated website. An important product of this work comes in the form of freely available simulation framework called gUPPEcore. It is designed for a wide range of situations in the field of extreme nonlinear optics, including bulk and structured media. A forward-looking feature of the framework is that it allows extensive user-defined customization. This framework is accompanied by gUPPElab, which is a collection of simulation templates designed to aid practical application. A dedicated website has been created to support new users of gUPPEcore/lab:

http://acms . arizona.edu/FemtoTheory/MK_personal/guppelab/.

\subsection{Broader impact}

Products of this effort open the door for computer-aided research and discovery in areas that traditionally relied on approximate methods, including nonlinear optics in highly multimode structures. Several method components developed in this effort are applicable in other fields, including computational quantum mechanics. The newly created approached also links two traditionally distinct communities of beam-propagation and nonlinear optics.

\subsection{Personnel Supported}

PI: Miroslav Kolesik

Postdoctoral Fellows

Jonathan Andreasen, Chen Xi (partial support), Paris Panagiotopoulos (partial support) 


\subsection{Brief summary of results concerning numerical methods}

- Derivation of the Generalized Unidirectional Pulse Propagation Equations (gUPPE). gUPPE provides a theoretical basis for a broad family of numerical models for simulation in the field of extreme nonlinear optics. Unlike its predecessor, the UPPE which is adequate for bulk media, the new theory was specifically designed to handle arbitrary geometries consisting of dispersive nonlinear materials.

Article: P1

- Design of a highly customizable framework, gUPPEcore, which provides all necessary infrastructure to implement various realizations of gUPPE. It provides a number of programming interfaces that allow for extremely flexible and, at the same time, simple definition and implementation of new capabilities. This approach ensures that the framework can "absorb" future developments in such fields as light-matter interaction modeling, and novel pulsed beam propagation algorithms.

Article: P1

Dedicated website facilitates software downloads and help new users.

- Design of an interface that serves to connect the gUPPEcore solver with the userdefined (or customized) implementations of arbitrary models for evaluation of the nonlinear medium response. This is a crucially important element of the core library. The early implementation of the interface was put to the test in a work utilizing a fully quantum mechanical, nonlinear polarization plug-in (P2). Several variations were tested throughout the duration of the project, with the goal to balance generality and ease of use. The final form of the interface was tested in a extensive study of the statistics of extreme events in optical light bullets (P10).

Papers P2, P10

- gUPPEcore propagator for hollow-core waveguides. The method resolves both the waveguide core and the cladding. Its important feature is that it captures correctly the dynamics of losses mediated by radiation through the cladding. In conventional methods, which rely on modal decomposition, these effects are only approximated. It was shown that full spatial resolution is necessary for quantitatively correct results. Paper P3

- Core-confined beam and pulse propagation method. This method allows the computational domain to be restricted solely to the core of a high-contrast waveguide or a leaky waveguide. The method is designed specifically for geometries with piece-wise constant material properties. It utilizes boundary conditions based on a "digital filter" that extrapolates the inner-domain optical field into the cladding by properly accounting for physical (differential) boundary conditions at material interfaces. Results show an order of magnitude performance improvement.

Paper P7

- High-performance, parallel gUPPE linear propagator plug-in based on the method of lines. Serves as a reference tool for validation and performance comparison of various 
approaches developed in this project.

Paper P6

- High-performance, parallel, gUPPE-based quantum Schrödinger equation simulator. This project tested and demonstrated the flexibility of the gUPPE framework in a setting outside of its native domain of nonlinear optics.

Paper P8

- Higher-order gUPPE propagator based on the alternating direction implicit method. Allows efficient simulation of fully space- and time-resolved pulsed-waveform simulations in strongly nonlinear situations. Its design "departs" from the fundamental mode, the chromatic properties of which are preserved exactly in this grid-based method. This novel approach marries advantages of the projection onto the fundamental mode, which underlies the generalized NLS method, and the space-resolving approach without the need to obtain higher-order modes of the structure in explicit form.

Paper S1

- Reflection-less computational domain boundary. A general method, applicable to pulsed beam propagation as well as to quantum-mechanics simulations. It uses a contour in the complex plane to represent each dimension of the numerical grid. It differs from previous methods (such as the complex coordinate stretching), in that arbitrary contours are admissible - even ones that exhibit sharp corners. The advantage of this approach is that transparent boundaries can be realized with a smaller number of grid points in the boundary layer. Moreover, the domain edge can "cut" into evanescent tails of guided modes. The result is a significantly smaller computational domain. This has been an important component for implementation of gUPPEcore propagators that are suitable for large-scale problems, such as supercontinuum generation on chips. Paper P12

\subsection{Physical results made possible by simulation capabilities de- veloped in this project}

This section presents a concise list of results, physical insights and sometimes unexpected observations that were obtained in various theoretical and/or experimental works, which utilized methods and software created in this project. It is meant to demonstrate that the simulation software developed is not only used to support and interpret experiments, but that it has become a tool for true computer-aided discovery.

The references pointing to published papers are classified and labeled as follows. In a number of projects, modeling required development work on gUPPEcore and various plugins as it relies upon, for example, light-matter interaction calculations. These works are labeled $\mathrm{P}$ and are enumerated in the corresponding list of publications, which acknowledge the support from this grant.

Other projects used the software, but did not require its modifications or improvements which would feed back into the development. Yet, these works provided a much needed 
testing grounds for gUPPEcore as it evolved over the course of several years. Such papers are labeled $\mathrm{M}$.

- Prediction and subsequent realization of externally refueled and dressed filaments. It is safe to say that the theoretical work that predicted dressed filaments would not be possible without the methods and software created by this project.

papers: M1, M2.

- Mid-infrared and longer wavelength nonlinear optics in gases differs qualitatively from the near-infrared and visible. Unlike the NLS, it is the nonlinearity that dominates over dispersion.

paper: M3.

- The above regime can result in pronounced carrier shocking and in extremely exotic pulsed waveforms. It will be interesting to understand how the traditional NLO changes when light-matter interactions, e.g., high-harmonic generation, are driven by such unconventional waveforms.

P3, M3, + a work under review

- Mid-infrared waveforms can be "tuned" in hollow waveguides such that amplitude, local frequency or both experience sudden, very strong change within a single cycle. Methods developed in this effort are required for quantitatively correct results.

P3

- Longer-wavelength filamentation relies on the until now un-appreciated mechanism of self-focusing collapse arrest through "temporal leakage of newly generated frequency components." The consequences are dramatic in terms of the filament properties. As of time of this writing, this work is being prepared for publication.

- Prediction of pronounced history-dependent effects that can occur in synthesized wavetrains. Surprisingly, drastically different ionization yields in low-intensity, and longduration pulse trains can be induced by controlling the relative timings of the most intense peaks.

M7

- Demonstration of a first-principle based, extremely economic description of nonlinear atomic response in terms of meta-stable states. gUPPEcore capabilities made a realistic-scale demonstration feasible.

S2

- The ubiquitous notion of the critical power for self-focusing lacks proper justification for ulrashort pulses. It has been shown that, in fact, that no sharp threshold exists separating the collapsing and regular solutions.

P4 
- Strong Raman-mediated frequency conversion in femtosecond Bessel beams. Extraextensive simulations were instrumental for the identification of the physical mechanism of bright on-axis emission.

M4

- Elucidated highly dynamic nature of self-focusing in nonlinear Airy beams and pulses. M5

- Demonstrated a surprising regime in nonlinear Airy pulsed beams in which free electron "plasma" causes an increase of the light intensity.

Being prepared for publication.

- Predicted that intense-pulse ionization produces excited and free-electron superpositions that respond as anisotropic systems due to coherent memory effects induced by the linearly polarized excitation pulse. This work is an example of application of the gUPPEcore simulation framework to a "non-native" (atomic physics) field

$\mathrm{P} 8$

- Elucidated spatial effects in high-harmonic radiation from the gas jet in a femtosecond enhancement cavity. This was a first ever simulation model encompassing a realistic scale in which all frequency components, from the fundamental to high harmonics and their interaction with atoms, were described by a quantum model.

$\mathrm{P} 2$

- Design of a practical accuracy-estimator of the uni-directional approximation (the assumption is necessary in all pulse propagation simulations)

M6

- Identified an until now un-recognized spatial effect which influences the location of characteristic spectral peaks that occur in supercontinua due to emission of dispersive waves. It was shown that these features, while being qualitatively reproduced by previous methods, can only be described accurately in simulations with full spatial resolution.

S1 


\section{Technical report}

\subsection{Theory of nonlinear pulse propagation in general structured media}

One of the motivations for this project was the prior absence of simulation methods that could handle difficult-to-model regimes in which rich spatial, temporal and spectral dynamics, together with the geometry of a waveguide structure play their roles simultaneously. The central outcome of this effort is the Generalized Unidirectional Pulse Propagation Equations (gUPPE) theoretical framework, which provides a most general basis for development of practical simulation methods specifically designed for these situations.

The gUPPE can be briefly summarized in a formal pair of coupled equations

$$
\begin{aligned}
\partial_{z} A_{\perp}^{F} & =\frac{+i}{2 \sqrt{\hat{L}}} e^{-i \sqrt{\hat{L}} z} \hat{N}_{\perp}\left[e^{+i \sqrt{\hat{L}} z} A^{F}+e^{-i \sqrt{\hat{L}} z} A^{B}\right] \\
\partial_{z} A_{\perp}^{B} & =\frac{-i}{2 \sqrt{\hat{L}}} e^{+i \sqrt{\hat{L}} z} \hat{N}_{\perp}\left[e^{+i \sqrt{\hat{L}} z} A^{F}+e^{-i \sqrt{\hat{L}} z} A^{B}\right]
\end{aligned}
$$

which describe forward- and backward-propagating components of the optical field, with $\hat{L}$ and $\hat{N}$ representing linear and nonlinear interaction operators, respectively. The linear operator $\hat{L}$ describes the geometry and constituent materials of the structure in which the optical pulse propagates,

$$
\hat{L} \vec{E}_{\perp} \equiv \frac{\omega^{2}}{c^{2}} \epsilon\left(r_{\perp}, \omega\right) \vec{E}_{\perp}+\Delta_{\perp} \vec{E}_{\perp}+\nabla \frac{1}{\epsilon} \vec{E}_{\perp} \cdot \nabla_{\perp} \epsilon
$$

where $\epsilon\left(r_{\perp}, \omega\right)$ encapsulates the geometry and dispersion properties of materials. The nonlinearity $\hat{N}$ can, in principle, be arbitrary.

It is an important feature of this system that linear and nonlinear interactions are separated, and that this separation is exact. In fact, the whole system above is free of approximations, and the sole assumption needed for its derivation is that the medium structures are nearly $z$-invariant. Since the derivation of these results is rather involved and requires a sequence of auxiliary systems which allow the passage from Maxwell's equations to this gUPPE pair, the reader should consult P1 for details.

The power of gUPPE lies in the marriage of the techniques of extreme nonlinear optics in bulk media one one hand, and many available beam-propagation computational methods for waveguides on the other. It thus opens a door to realistic numerical modeling of systems that were, until recently, out of reach. (S1)

Significant portions of this effort were dedicated to development, implementation, and applications of the gUPPE theory to concrete systems. The experience thus gained in turn guided the work on the freely available software, gUPPEcore, which implements the framework. The authors believe that in time it will become a de-facto standard simulation tool for extreme nonlinear optics. 
The following figure shows an example of a fully resolved simulation of a high-power pulse propagating in a hollow waveguide. The gUPPE approach made it possible to capture the optical field across the core, where nonlinear interactions occur, and in the cladding, which crucially affects the propagation properties of the waveform.
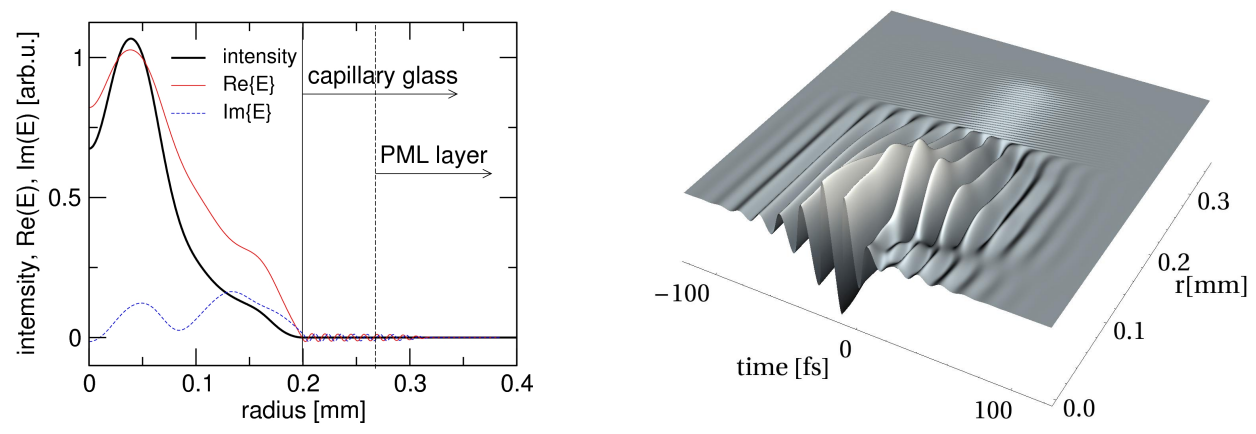

Figure 1. Fully resolved simulation of a high-power pulse in a hollow waveguide. Left: Crosssection of a fully resolved optical field. Right: Space-time profile of the electric field waveform. The diagonal structure in the upper portion of the figure is the correctly computed emitted energy. Failure to model this loss channel negatively affects the simulated pulse intensity, and through it the interaction with the gas in the capillary bore.

It should be noted that while the traditional methods applied to this and similar systems do capture many qualitative features of the underlying physics, it has been shown that the approximate results are not correct in the quantitative sense. The gUPPE approach thus offers a correct, yet computationally feasible way to model many important situations, including, for example, nonlinear pulse propagation in high-pressure waveguides.

\subsection{New simulation methods for NLO in high-contrast waveguides}

In practical nonlinear-optics (NLO) applications, one restricts the coupled forward-backward system to a single equation. This sole approximation, which is not new and has been a tacit assumption in all one-way propagation theories, assumes that the nonlinear interaction caused by the backward waves is negligible. Consequently if the backward ave $A^{B}$ vanished initially, gUPPE reduces to a single equation for $A^{F}$.

The central task in the application of gUPPE to a concrete system lies in the design and implementation of the linear propagator $\exp [+i \sqrt{\hat{L}} z]$. This is, admittedly, an "intimidating" operator, and one could ask if anything was gained at all by reformulating the Maxwell system into one that requires implementation of such a propagator. Fortunately, homogeneous media admit exact, namely spectral methods, and for structured media one can utilize any of the beam-propagation approaches available. Several new methods were developed in this project, and they are described next. 


\subsubsection{Core-confined beam propagation method for guiding and leaky structures.}

The core-confined beam propagation method was developed as a linear propagator for the gUPPE framework. However, it is a generally useful approach targeting applications with high-contrast waveguides, as exemplified in this figure:
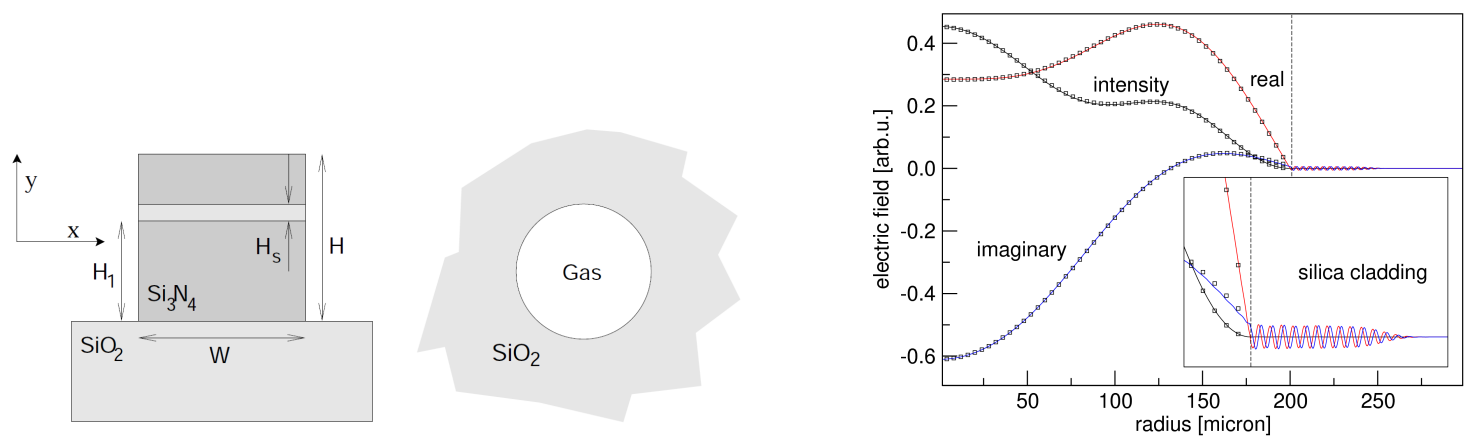

Figure 2. Illustration of the core-confined beam-propagation method. Left: Typical application scenarios include complex integrated waveguides (left), e.g., for on-chip supercontinuum generation, and capillaries (right), which are leaky or lossy waveguides. Right: The coreconfined approach makes it possible to restrict the computational domain solely to the core (symbols). Yet it correctly and accurately captures complex and dynamic shapes of the propagating pulse (solid lines).

The approach was demonstrated and verified against traditional BPM methods. It was shown to be highly accurate, and capable of correctly capturing multi-mode dynamics that arise when the light coupled into a waveguide does not perfectly match the fundamental guided mode. Importantly, for applications in nonlinear optics, this algorithm produces more than an order of magnitude speed up when compared to the method that requires grid sampling over the waveguide cladding.

\subsubsection{Efficient simulation of pulse propagation in high-contrast nonlinear nanowaveguides}

Yet another linear propagator implemented in this project for the gUPPE framework is using the Method of Lines (MOL) beam-propagation approach. This was utilized to demonstrate what is the first truly wide-band, space- and time-resolved simulation of supercontinuum generation on a chip. Two methods were designed, one encompassing the waveguide core plus cladding, the other implementing the core-confined method described above. The following picture illustrates the simulated spectra: 

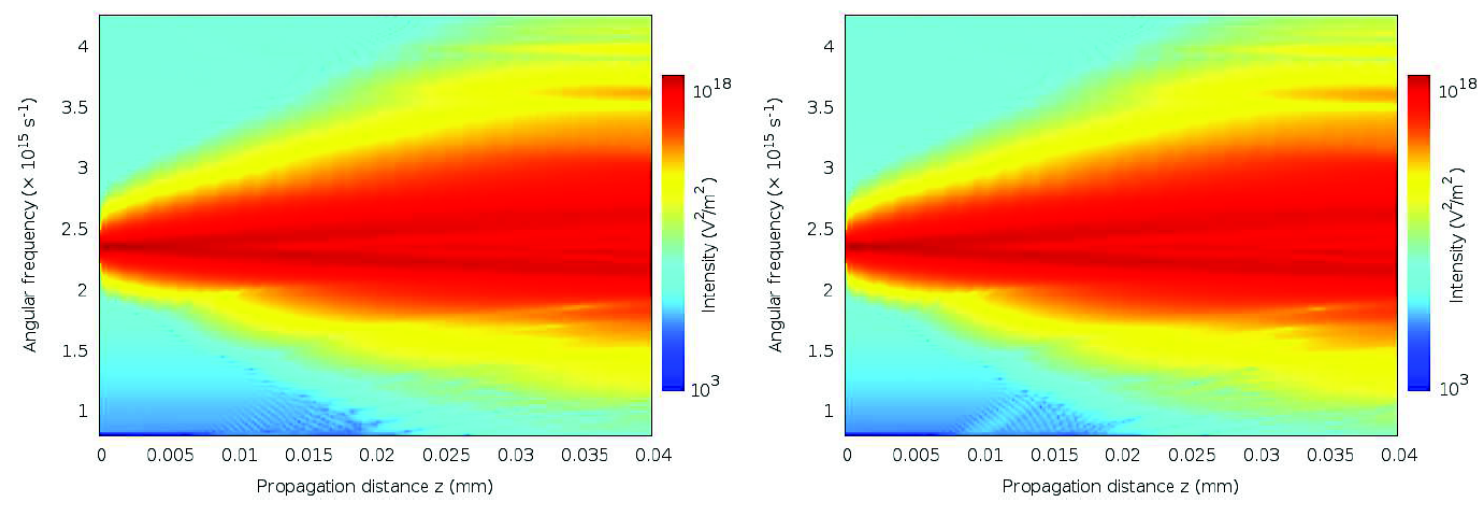

Space- and time-resolved, $3+1$ dimensional simulation of supercontinuum generation in a highcontrast waveguide. Two different algorithms are compared and verified here. Spectral power is shown in log scale versus the propagation distance. Left: The fully resolved MOL propagator.

Right: The order-of-magnitude faster core-confined propagator.

The above maps of the spectral power show the evolution of the supercontinuum in the waveguide. Obviously, what is shown is extremely good agreement over a large dynamic range of six orders of magnitude, and thus validates the core-confined approach.

\subsubsection{New transparent-boundary method}

Simulation of space-time dynamics of femtosecond pulses are large-scale by default. It is therefore crucial to be able to keep the computational domains as small as possible, which was the rationale behind the development of a new type of transparent boundary condition. It is a generalization of the Perfectly Matched Layer (PML) method designed with the gUPPE propagators in mind. However, the method is completely general, and applicable without modifications to any BPM approach. The idea is illustrated in the following figure:

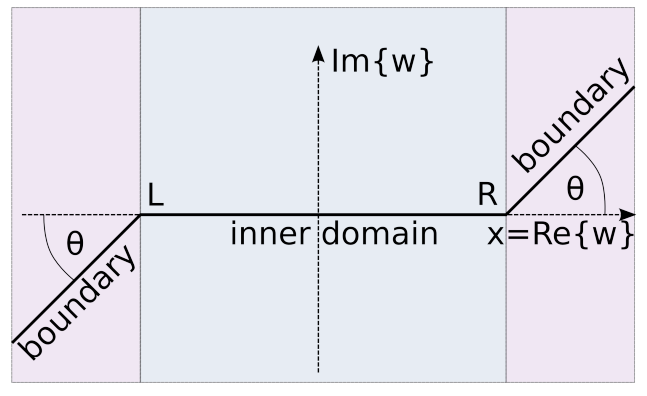

One-dimensional computational domain supported by a contour (thick line) in the complex plane $w,(\operatorname{Re}\{w\}=x)$. Real axis corresponds to the original simulation domain, that is now diverted into upper (right) and lower (left) complex half-planes. Outgoing waves decay exponentially toward both ends of the contour. Angle $\theta$ can arbitrary, including $\Theta=\pi / 2$.

The novelty of this method is that by taking advantage of the analytic properties of the simulated solutions, it can "map" the computational domain onto arbitrary contours in the complex plane. This includes contours with sharp corners, which in turn eliminates the need for a smooth transition between the inner domain and the perfectly matched layer. As 
a result, the transparent boundary layer can be extremely thin while preserving very low residual reflectivity. This is illustrated as follows:

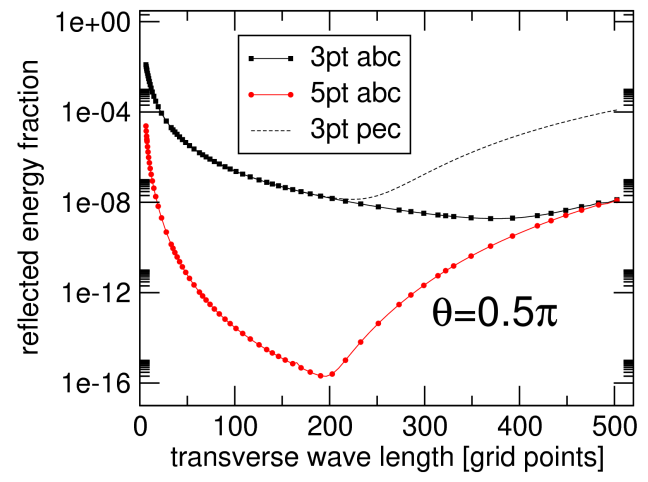

Residual grid-edge reflectivity for $\Theta=\pi / 2$, when the domain contour (see previous figure) departs from the real axis at a right angle. Sets with symbols represent boundary layer terminated with a local absorbing boundary condition (abc). Dashed line was obtained with a vanishing boundary condition (pec). The reflectivity remains very low even for boundary layers consisting of only five points.

This method can be coupled with almost all implementations of gUPPE propagators that use finite-difference discretization. It has been utilized to a great effect in our latest model of the supercontinuum generation on a chip.

Note that the new transparent-layer applications are possible beyond optical beam and pulse propagation modeling. The method applies equally to quantum simulations problem, and in fact it has already been in heavy use in our AFOSR supported research on firstprinciple nonlinear response theory (FA9550-13-1-0228).

\subsubsection{Higher-order methods for supercontinuum generation on chip}

Recently there has been interest and much experimental and theoretical work concerning supercontinuum generation on chips. Because full scale simulations have not been feasible so far, approximate, mode-projection methods have been used to model and interpret experiments. In this project, a method has been developed that for the first time makes it possible to simulate the physics of on-chip supercontinuum generation with full resolution. Importantly, the new approach requires relatively modest computing effort. Moreover, this application brought new insights into the underlying dynamics, which in turn opens further possibilities for development of even more efficient simulation approaches.

To implement the gUPPE propagator, one designs a method that builds on the success of the traditional methods that project the optical field onto the fundamental mode of the waveguide. Specifically, the propagator can be expressed as

$$
e^{+i \sqrt{L} z}=e^{i \beta z} e^{i \beta z\left[\sqrt{1+\frac{L-\beta^{2}}{\beta^{2}}}-1\right]} \approx e^{i \beta z} \exp \left[\frac{z}{2 \beta}\left(L-\beta^{2}\right)\right],
$$

which numerically reproduces the chromatic properties of the fundamental mode exactly. Higher-order mode dynamics is accounted for implicitly through the fourth-order accurate finite-difference discretization. The new method is coupled with the new transparent boundary conditions mentioned earlier. Together with the high order of discretization accuracy, it allows use of a relatively small domain: 


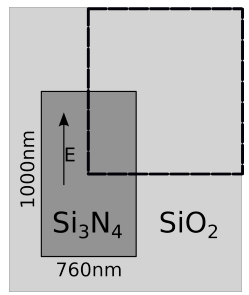

Simulated waveguide. Symmetric solution is assumed, so that the computational domain can be restricted to the rectangle indicated in the picture. New transparent boundaries allow the grid edge to be closer to the core. As a result, the spatial discretization only requires a few hundred grid points.

With a few hundred grid points in the spatial cross-section of the computational domain, the numerical complexity of what was a truly large-scale simulation decreases to an acceptable level. Realistic simulations only take a few hours on common computing hardware.

The following figure shows a comparative example of simulated supercontinuum spectra.
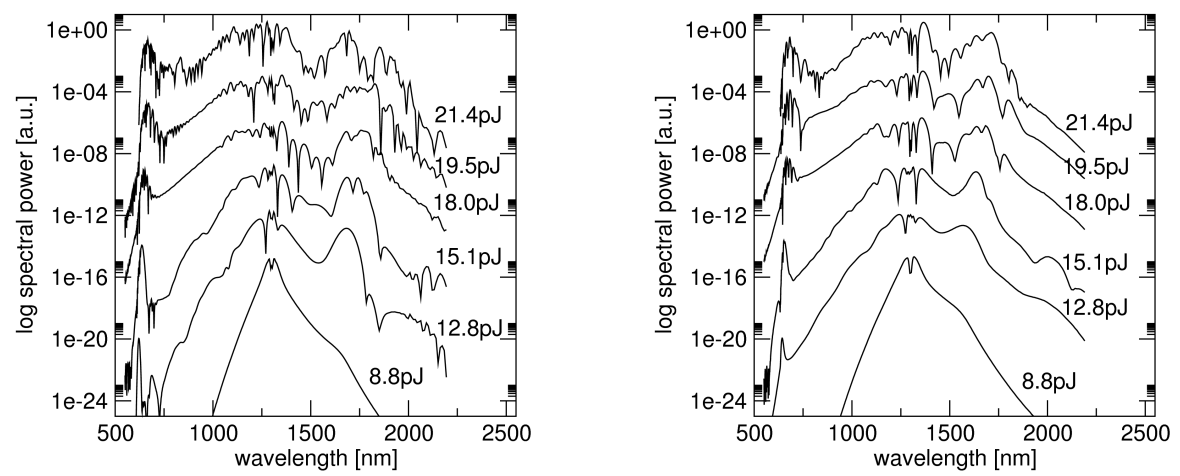

Supercontinuum generation in a waveguide after $40 \mathrm{~mm}$ propagation. Left: Traditional method, using Generalized Nonlinear Schrödinger Equation with the projection on the fundamental mode. Right: gUPPE simulation with full spatial resolution. Labels indicate output pulse energies.

The supercontinuum spectra exhibit characteristic short- and long-wavelength dispersive wave features. The next figure shows that the approximate method produces the dispersive wave peaks at shifted locations.
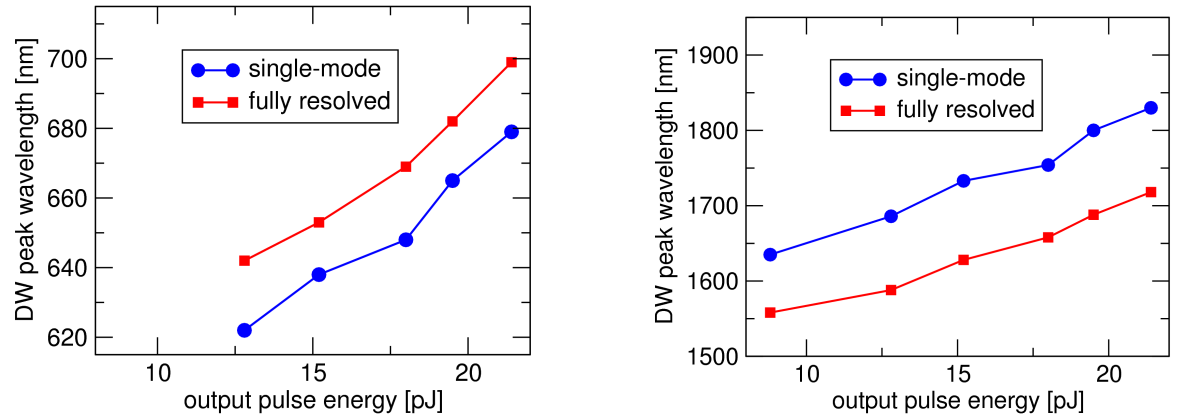

Dispersive-wave spectral peak locations as functions of pulse energy for the short wavelength peak (left) and the long-wavelength side of the spectrum (right).

Further analysis identified a new spatial effect that adds to the spectral shift of dispersivewave peaks. Similar to the intensity-dependent modification of a phase-matching relation 
that relates the pulse central wavelength to that of the dispersive wave, this effect modifies (narrows) the transverse shape and through it also the effective dispersion of the nonlinearly propagating wavepacket. Importantly, only a fully resolved simulation can capture these dynamics. Thus, by pushing down the computational complexity of the underlying simulations, this new approach results in a more realistic model, which brings better understanding of supercontinuum generation dynamics in confined geometries.

\subsubsection{Large-scale simulations and parallel performance}

Significant work went into achieving efficient parallelization. Because gUPPEcore is meant to be utilized by users working on very different topics, from the nonlinear fiber optics to Tera-Watt laser physics, large "dynamics range" in applications is a must. In other words gUPPEcore performs efficiently in a tiny simulation of pulse propagating in a nonlinear waveguide, as well as in a large-scale modeling of a turbulent pulsed beam using up to two billion variables.

In terms of hardware, gUPPEcore mainly targets modern desk-side workstations with up to sixteen CPU cores. However, smaller problems can be tackled efficiently on a laptop, and the big ones can utilize clusters with hundreds of cores:
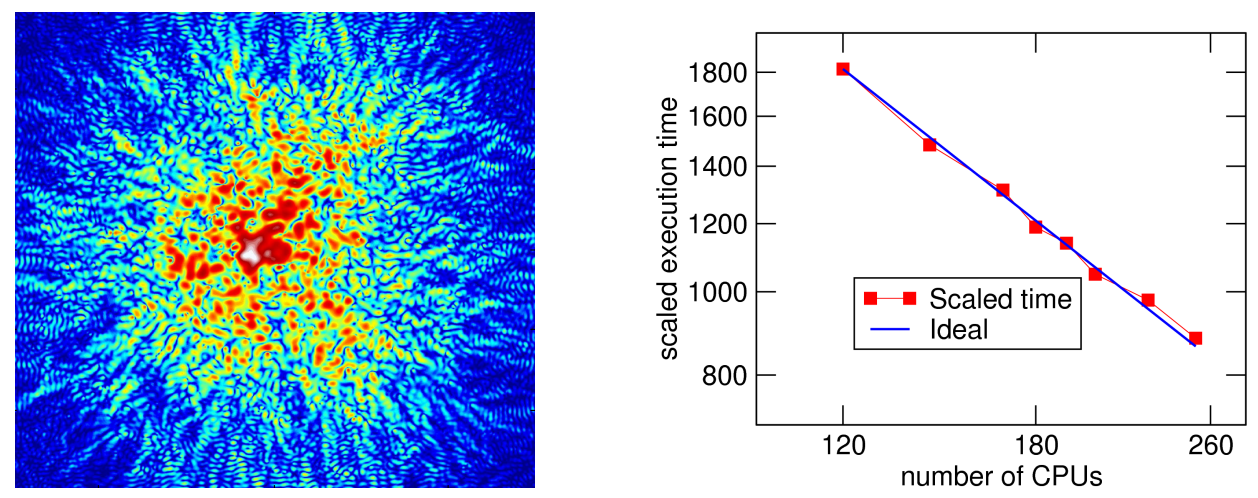

Large-scale simulation and parallel performance example. Left: Anderson localization of light. Pulsed beam propagates through a medium with a weak refractive-index disorder, until its diffraction is arrested by the localization. The picture shows the "random" intensity profile on the $\log$ scale over an area of $6 \times 6 \mathrm{~mm}$. Five hundred million variables are used to represent this pulsed waveform. To simulate the interplay with nonlinearity, up to two billion degrees of freedom are needed. The almost-ideal parallel scaling with up to 256 processors in use is shown in the right hand side panel.

Two parallelization paradigms were tested throughout the duration of the project. The first was a mixed approach, with Pthreads taking advantage of the local shared memory on a cluster node, while using MPI to communicate between nodes. The second approach is purely MPI-based, and was adopted in the final version of the software as it gives superior performance in a vast majority of simulation scenarios. Several strategies of domain decomposition for parallelization were tested as well. The final version works with a linear-graph domain decomposition, but utilizes two alternating "views" designed such that the implementation 
of other simulator components is completely isolated from the parallelization issues. For example, a linear gUPPEcore propagator can be implemented with a beam-propagation algorithm without any need for, or reference, to parallel operations. This design is crucial for simplification of user-defined customizations, which are coded as if for a single processor.

\section{3 gUPPEcore and gUPPElab software}

One of the objectives of this work was to create practically useful implementations. gUPPEcore/lab are the results of this effort.

\subsection{1 gUPPEcore}

gUPPEcore is a library of core capabilities that collectively implements the gUPPE theoretical framework. It was designed as a free software available to interested research groups but also suitable for teaching and education purposes. The vision guiding the gUPPEcore development was to keep it as lean as possible, while making customization straightforward. This is why significant volume of work went into its design, re-design and testing such that the result be as future-proof as possible. In particular, gUPPEcore has been made immune to the inevitable progress in light-matter interaction theory and modeling. It has the capability to adopt a very broad class of nonlinear response plug-ins, which gives the user complete freedom to decide what physics and what models control his/her simulation.

gUPPEcore handles other key components in a similar way. For example, initial pulse conditions can be completely customized. It is also possible to introduce user-defined addons which implement various operations over the simulated optical fields, such as optical filters, axicons, lenses, apertures, etc. Importantly, gUPPEcore is built such that these additions do not require expert programmers. Rather, the "typical user" envisioned during the development was an experimentalist graduate student who needs to perform simulations, but must not spend excessive time developing software.

An important forward-looking feature in gUPPEcore is its interface that allows userdefined geometry and linear propagators. While it admittedly requires some experience in numerical methods, this capability keeps gUPPEcore open to the plethora of beampropagation methods developed by various communities. In principle, any of them can be implemented into the linear propagator in gUPPEcore.

\section{gUPPEcore capabilities at a glance:}

- Carrier-resolving Maxwell simulator for pulses with a well-defined propagation direction

- Customizable geometry and optical field representation of the transverse cross-section of the pulsed beam.

- Pulse durations from picoseconds down to single-cycle

- Robust simulation of extremely nonlinear regimes resulting in ultra-broad spectra and sharp temporal waveforms. 
- Family of robust spectral propagators included

- Fiber, waveguide, and bulk-media geometries

- Isotropic and anisotropic dispersive media

- Common scenarios typical of optical filamentation do not require any customization

- Provides interfaces for user-defined:

1. Initial conditions describing pulsed beams

2. Operations representing optical elements such as lenses, apertures, and filters

3. Models of light-matter interactions

4. Transverse cross-section geometry of the waveguide and the corresponding linear propagator

- MPI-based parallelization allows up to a few billions of degrees of freedom to represent the optical pulse

\subsection{2 gUPPElab}

\section{Software components:}

1. Core libraries for Linux and OSX systems and pre-compiled executables

2. Header files and sources for the user-addon development and customization

3. Source files with examples/templates for user-defined initial conditions, wave-form modifying operators, and nonlinear medium-response models

4. A library of worked-out examples and simulation templates.

gUPPElab is first and foremost a collection of program sources and worked-out examples. Its purpose is two-fold. First, it serves as a tutorial for new gUPPEcore users. The least painful way to learn a new software is often by way of example. This is the philosophy adopted for the lab. Moreover, the tutorial examples also serve as a convenient point of departure for new simulations. The collection of exercises is chosen such that it covers the most relevant scenarios, which can be easily modified or customized to different purposes.

The second role of gUPPElab is to provide templates for user-defined add-ons. The templates are simple, fully functional examples with explained source codes. It is a relatively simple matter for a new user to follow the customization scheme. In most cases it will only require writing small portions of simple code, which does not require extensive programming experience. 
The typical simulation template consists of a README.pdf file, a set of functional input files that define one or more simulations, sample outputs, and scripts that illustrate the execution of the simulation and extraction of observable quantities from the simulator outputs. The README.pdf contains the description of the task in the given exercise. It identifies which elements of the gUPPEcore usage are described, what is the physical context of the simulation example, details the included input files for simulations, and suggests the work flow. Having less experienced users in mind, these examples are used to illustrate both the capabilities of the simulation framework and potential pitfalls.

The concept of the lab was tested by the PI both in summer schools and through collaborations with several research groups in which the simulation software was used. The selection of examples included in the lab strives to find an optimum between keeping this "tutorial set" as concise as possible, and covering the breadth and depth of the gUPPEcore simulation framework capabilities.

\subsection{3 gUPPElab/core website}

A dedicated website: http://acms.arizona.edu/FemtoTheory/MK_personal/guppelab/has been set up to facilitate downloads of the gUPPElab/core software. It is envisioned that in the future this website will also serve as an exchange for source-codes implementing various user-defined capabilities and additions to the gUPPEcore library. Authors hope that this will help to create a community of users, and that gUPPEcore will become a standard for modern numerical methods in the field of extreme nonlinear optics.

\subsection{Collaborations}

Collaborations were crucial for this effort. As one of the projected outcomes of this project was in applications of the newly developed capabilities to relevant problems in the general field of nonlinear optics, development versions of the software were shared with several research groups. These include the groups of D. Faccio at Heriot Watt, D. Christodoulides at CREOL, Ch. Durfee at Colorado School of Mines, and more recently the group of D. Lorenc of the International Laser Center in Bratislava, Slovakia. Extremely important and fruitful was our close connection with the ACMS MURI project, FA9550-10-1-0561. A number of MURI works benefited by using this project's software, and provided precious feedback for its development. 


\section{Peer-Reviewed Journal Articles}

\subsection{Peer-reviewed publications acknowledging the support under this project}

P1 J. Andreasen and M. Kolesik:

Nonlinear propagation of light in structured media: Generalized unidirectional pulse propagation equations.

Physical Review E, 86(3, 2) (2012) 036706.

P2 M. Kolesik, E. M. Wright, J. Andreasen, J. M. Brown, D. R. Carlson, and R. J. Jones: Space-time resolved simulation of femtosecond nonlinear light-matter interactions using a holistic quantum atomic model: Application to near-threshold harmonics.

Optics Express, 20(14) (2012) 16113-16128.

P3 J. Andreasen and M. Kolesik:

Mid-infrared femtosecond laser pulse filamentation in hollow waveguides: A comparison of simulation methods.

Physical Review E, 87(5) (2013) 053303.

P4 P. Polynkin and M. Kolesik:

Critical power for self-focusing in the case of ultrashort laser pulses.

Physical Review A, 87(5) (2013) 053829.

P5 X. Chen, P. Polynkin, and M. Kolesik:

Raman effect in self-focusing of few-cycle laser pulses in air.

Optics Letters, 38(12) (2013) 2017-2019.

P6 J. Andreasen and M. Kolesik

Efficient simulation of unidirectional pulse propagation in high-contrast nonlinear nanowaveguides .

Nanoscale Systems: Mathematical Modeling, Theory and Applications 2 (2013) 157165.

P7 J. Andreasen and M. Kolesik:

Core-Confined Beam Propagation Method for Guiding and Leaky Structures.

Journal of Lightwave Technology, 31(18) (2013) 2999-3005.

P8 J. Andreasen, E. M. Wright, and M. Kolesik:

Optical Response of Atomic Gases to Ultrafast Pump-Probe Pulses.

IEEE Journal of Quantum Electronics, 49(12) (2013) 1088-1096.

P9 M. Kolesik and J. V. Moloney:

Modeling and simulation techniques in extreme nonlinear optics of gaseous and condensed media.

Reports on Progress in Physics, 77(1) (2014) 016401. 
P10 T. Roger, D. Majus, G. Tamosauskas, P. Panagiotopulous, M. Kolesik,G. Genty, A. Dubietis, D. Faccio:

Extreme Events in Resonant Radiation from Three-dimensional Light Bullets.

Physical Review A, in press.

P10 M. Kolesik, J. M. Brown, K. Schuh, S. W. Koch, J. V. Moloney:

Ultrafast light-matter coupling in condensed and gaseous nonlinear media.

Proceedings of SPIE, ISBN: 9781628412130, paper 9186-15, in press

P12 A. Bahl, A. Teleki, P. Jakobsen, E. M. Wright, M. Kolesik:

Reflectionless beam propagation on a piecewise linear complex domain

Journal of Lightwave Technology, in press.

\subsection{Under-review publications acknowledging the support under this project}

As of time of this writing, the following papers are going through the review process.

S1 J. Andreasen, A. Bahl, M. KolesikJ. Andreasen:

Spatial effects in supercontinuum generation in waveguides.

Optics Express, in review.

S2 M. Kolesik, J.M. Brown, A. Teleki, P. Jakobsen, J.V. Moloney, E.M. Wright:

Meta-stable electronic states and nonlinear response for high-intensity optical pulses. Optica, in review.

\subsection{Peer-reviewed works utilizing software developed in this project}

The following papers (which all acknowledge the support from the ACMS MURI project FA9550-10-1-0561) benefited from the connection to the present project through significant use of the simulation software developed in the present project.

M1 M. Scheller, M. S. Mills, Mohammad-Ali Miri, W. Cheng, J. V. Moloney, M. Kolesik, P. Polynkin, D.N. Christodoulides:

Externally refuelled optical filaments

Nature Photonics 8 (2014) 297-301.

M2 M. S. Mills, M. Kolesik, and D. N. Christodoulides:

Dressed optical filaments.

Optics Letters, 38(1) (2013) 25-27. 
M3 P. Whalen, P. Panagiotopoulos, M. Kolesik, J.V. Moloney: Extreme carrier shocking of intense long-wavelength pulses. Physical Review A, 89(2) (2014) 023850.

M4 M. Scheller, Chen Xi, O. Ariunbold Gombojav, N. Born, J.V. Moloney, M. Kolesik, P. Polynkin: Raman conversion in intense femtosecond Bessel beams in air.

Physical Review A, 89(5) (2014) 053805.

M5 C. Ament, M. Kolesik, J. V. Moloney, and P. Polynkin: Self-focusing dynamics of ultraintense accelerating Airy waveforms in water. Physical Review A, 86(4) (2012) 043842.

M6 M. Kolesik, P. Jakobsen, and J. V. Moloney: Quantifying the limits of unidirectional ultrashort optical pulse propagation. Physical Review A, 86(3) (2012) 035801.

M7 M. Kolesik, J. M. Brown, J. V. Moloney, D. Faccio: History-dependent effects in subcycle-waveform strong-field ionization. Physical Review A, in press.

\section{Dissemination and Outreach}

\subsection{Colloquiua, invited talks, and keynote lectures delivered by PI}

These presentations included theory, algorithm description(s), and/or implementations in software developed in this project.

L1 Invited: Pulse propagation and light-matter interaction in filamentation COFIL2014: The 5th International Symposium on Filamentation 09/21/2014, Shanghai, China

L2 Invited: Ultrafast Light-Matter Coupling in Condensed and Gaseous Nonlinear Media SPIE Optics+Photonics 08/19/2014, San Diego, U.S.A.

L3 Invited: Pulse propagation and non-perturbative light-matter interaction in extreme nonlinear optics

"The nonlinear meeting," Heriot Watt University

05/21/2014, Edinburgh, UK

L4 Invited: Mathematical Methods and Models in Laser Filamentation"

International Workshop on: "Mathematical Methods and Models in Laser Filamentation"

03/11/2014, Montreal, Canada 
L5 Keynote lecture: Light-matter interactions at ultra-fast time-scales and high intensities: modeling and simulation perspective

Max Planck Institute for the Science of Light Workshop 06/24/13 - 06/28/13, Gössweinstein, Germany

L6 Invited: Current Trends and Challenges in femtosecond Laser Filamentation Modeling and Simulations

American Mathematical Society Meeting on

"Mathematics of Optical Pulse Propagation: Modeling, Analysis and Simulations" 10/27/12 - 10/28/12, Tucson, AZ

L7 Invited: Toward self-consistent models of non-linear optical response in femtosecond filaments

COFIL 2012, 4th International Symposium on Filamentation 10/07/12 - 10/12/12, Tucson, AZ

L8 Invited: Toward self-consistent models of non-linear optical response in femtosecond filamentation

International Laser Physics Workshop

07/23/12 - 07/27/12, Calgary, Canada

L9 Invited: Holistic approach to models of non-linear optical response on femtosecond timescales

International Workshop on Laser-Matter Interaction

06/25/12 - 06/29/12, Porquerolles, France

L10 Colloquium: Extreme nonlinear optics: A computational perspective

College of Optical Sciences, Colloquium series

04/05/2012, Tucson, AZ

\subsection{Summer-school lectures and short courses delivered by PI}

The following courses covered the theory, and and/or its implementations in software developed with this project support.

S1 Cork School 2013:

I. UPPEcore Simulator design and usage, Simulation examples

II. Nonlinear pulse-propagation in waveguding structures

III. Light-matter interaction modeling beyond the current model

School on theory and mathematics modeling of ultrashort pulse propagation 08/28/13 - 08/02/13, University College Cork, Ireland

S2 ACMS MURI School 2012:

Computational Methods for Nonlinear PDEs describing Ultrashort Optical Pulses 
School on theory and mathematics modeling of ultrashort pulse propagation 03/18/12 - 03/20/12, University of Arizona, Tucson AZ

S3 DEPS topical meeting, Advanced High-Power lasers: Ultrashort Pulse Laser Induced Filaments

Short course \# 7, delivered jointly with H. Milchberg 06/11/2012, Broomfield, CO 